\title{
Estrategia de proyección del turismo de eventos e incentivos en la zona central de Cuba.
}

\section{Projection strategy of tourism events and incentives in the central zone of cuba.}

\author{
Lic. Kathleen B. Taboada Vega., ${ }^{1} \&$ MSc. Luis Miguel Campos Cardoso. ${ }^{2}$
}

\section{Resumen.}

El Turismo de Eventos e Incentivos se presenta en la actualidad como una alternativa tentadora, reportando significativos ingresos, contribuyendo a disminuir los efectos de la estacionalidad y reportando una amplia gama de beneficios comerciales, todo lo cual ha condicionado un crecimiento paulatino. En este sentido, Cuba cuenta con importantes potencialidades para hacer frente a este mercado. La zona central del país, se presenta hoy como un destino consolidado en algunas modalidades, sin embargo, a pesar de contar con elementos favorables para el mercado de eventos, no ha sido consecuente el resultado obtenido en los últimos años. Es así que se desarrolló la investigación, enfocada a lograr una proyección estratégica del turismo de eventos e incentivos en las provincias de Villa Clara, Cienfuegos y Sancti Spíritus. De tal manera se partió de un análisis bibliográfico, que permitió sentar las bases teórico-metodológicas requeridas y la decantación por los procedimientos de Bermúdez (2013) y Peñalver (2015), ambos científicamente probados, y dirigidos, respectivamente, al diagnóstico y proyección estratégica del Turismo de Eventos e Incentivos; deviniendo en un conjunto de objetivos y acciones estratégicas por Áreas de Resultados Claves, dirigidas a la solución de la problemática identificada. Para esto fueron utilizados métodos y técnicas tales como la observación participante, revisión documental, procesamiento de encuestas y entrevistas no estructuradas.

Palabras claves: destino turístico, turismo, turismo de eventos e incentivos, eventos, gestión de eventos.

\section{Abstract.}

The Tourism of Events and Incentives is currently presented as a tempting alternative, reporting significant revenues, contributing to diminish the effects of seasonality and reporting a wide range of commercial benefits, all of which have conditioned a gradual

\footnotetext{
${ }^{1}$ Universidad de La Habana, Facultad de Turismo. La Habana, Cuba. kathleen.taboada@ftur.uh.cu

${ }^{2}$ Universidad de La Habana, Facultad de Turismo. La Habana, Cuba.
} 
growth. In this regard, Cuba has significant potential to face this market. The central zone of the country, is presented today as a consolidated destination in some modalities, however, despite having favorable elements for the event market, the result obtained in recent years has not been consistent. Thus, the research was developed, focused on achieving a strategic projection of tourism events and incentives in the provinces of Villa Clara, Cienfuegos and Sancti Spíritus. In this way, it was based on a bibliographic analysis, which allowed to establish the theoretical-methodological bases required and the decanting by the procedures of Bermúdez (2013) and Peñalver (2015), both scientifically proven, and directed, respectively, to the diagnosis and strategic projection. of Event Tourism and Incentives; becoming a set of objectives and strategic actions by Key Results Areas, aimed at solving the identified problem. For this, methods and techniques such as participant observation, documentary review, survey processing and unstructured interviews were used.

Keywords: tourist destination, tourism, event and incentive tourism, events, event management.

\section{Introducción}

El sector turístico se ha convertido en una de las mayores fuentes generadoras de empleo, de divisas y de estímulo a la inversión y al crecimiento económico. Solamente en el año 2016, según el último número del Barómetro de la Organización Mundial del Turismo (OMT) para el turismo global, las llegadas de turistas internacionales aumentaron un 3,9 $\%$ hasta situarse en los 1.235 millones, alrededor de 46 millones de turistas más que el año anterior y superando así en 300 millones la cifra record alcanzada en 2008 antes de la crisis económica (OMT, 2016).

Sin embargo, un nuevo turismo, definido y especializado ha surgido en años recientes y se ha convertido en una de las ramas más importantes de los viajes internacionales por su volumen y por la derrama de divisas que representa: el turismo de eventos.

ICCA (International Congress and Convention Association), la principal asociación mundial de turismo de reuniones o MICE (meetings, incentives, conventions, exhibitions), registró en 2015 la cifra record de más de 12.000 reuniones asociativas (571 más que en 2014), lo que confirma el buen crecimiento del sector. Cabe destacar que aun cuando el crecimiento de los eventos demuestra ser imparable, se ha producido un descenso en las cifras de reuniones y congresos efectuados en los países del Top 10 de ICCA, lo cual indica que se están distribuyendo más uniformemente, entre un mayor número de destinos internacionales (ICCA, 2017).

La sociedad ha evolucionado, el consumidor turístico ya no es lo que era. De modo tal, que hablar de la modalidad en su estado puro actualmente, es una hipótesis inalcanzable. El evento es solo la pieza central de un enorme rompecabezas, donde cada ficha juega su rol, en pos de convencer y enamorar a un, cada vez más, difícil consumidor. Entonces, se habla de la modalidad, e implícito se encuentran numerosos actores, donde los participantes y las sedes están lejos de ser los únicos (Morales, 2014).

Aun cuando Cuba es un país que se pensaría idílico para la realización de eventos que respondan a su marcado reconocimiento internacional en el ámbito de la ciencia, la técnica y la cultura, o simplemente a lo cautivador de su esencia caribeña, la explotación 
de la modalidad ha sido insuficiente atendiendo a las potencialidades reales con que cuenta. No obstante, algunas instituciones de los subsistemas exógeno y endógeno del sistema turístico buscan hoy una proyección hacia la demanda de eventos.

La zona central del país, cuenta por excelencia con una de las mayores capacidades habitacionales de la isla, al norte de la provincia de Villa Clara, marcadas por el confort, accesibilidad y un entorno paradisíaco. Estos factores, en conjunto con otras muchas variables, han sido determinantes para la selección de estos hoteles como sedes de eventos.

Las propias características favorables del destino y la existencia de una infraestructura, servicios y equipamiento necesarios lo hacen altamente atractivo para el desarrollo de eventos lo cual contribuiría notoriamente a mitigar la estacionalidad turística.

Sin embargo, aún no se han logrado los niveles de profesionalidad requeridos, pues no se ha alcanzado la total integración del producto turístico por lo que no se han conseguido cuotas significativas de otros segmentos de mercado, además del de sol y playa, ni el máximo aprovechamiento de todo el potencial del destino para el desarrollo del turismo de eventos.

La situación de la actividad en el país tiene repercusión en el destino, viéndose afectada en un primer orden, por la inexistencia de un organismo rector encargado de gestionarla y la eliminación de cursos formadores de Organizadores Profesionales de Congresos.

Tampoco se tienen en cuenta análisis, planes, estrategias y documentos que direccionen la modalidad. A su vez, se adolece de registros, estadísticas e informaciones que ilustren el desarrollo del Turismo de Eventos e Incentivos y se carece de herramientas que permitan su gestión eficiente, teniendo en cuenta el carácter integrado de la oferta en el destino, la demanda, la competencia, el entorno, las potencialidades e indicadores del turismo de eventos e incentivos.

El tema seleccionado deriva de una necesidad corroborada en la práctica, la cual establece la condicionalidad de solucionar problemas inducidos por debilidades en la gestión de eventos e incentivos por la existencia de debilidades en la coordinación de acciones estratégicas y su proyección integral, encaminadas a convertir a la zona central del país en un verdadero destino de esta modalidad.

Es así que se plantea como objetivo general: Diseñar una estrategia de proyección del turismo de Eventos e Incentivos para la zona central de Cuba que fortalezca la gestión de esta modalidad.

Por otra parte, el resultado de la investigación desarrollada tributa a la actualización de los Lineamientos de la Política Económica y Social del Partido y la Revolución para el período 2016-2021 del séptimo Congreso del Partido, y los lineamientos de la proyección estratégica del Ministerio de Turismo (MINTUR) que establecen la necesidad de diversificar la oferta e incrementar la promoción de Cuba como destino de eventos, además de constituir una fuente alternativa de captación de divisas para el país. 


\section{Desarrollo}

Para el logro de una proyección estratégica del destino, y teniendo en cuenta las características del mismo y la integración holística que se quiere alcanzar se adoptó el procedimiento aportado por Peñalver, 2013: Procedimiento para la gestión del Turismo de Eventos e Incentivos en un destino Turístico.

El procedimiento consta de 5 Fases: en primer lugar la Organizativa, donde se organiza el equipo de trabajo; luego la Analítica cuyo objetivo es el diagnóstico de la gestión del turismo de eventos e incentivos en el destino; le sigue la Estratégica que pretende la definición de la estrategia general de gestión del turismo de eventos e incentivos en el destino y el planteamiento de objetivos estratégicos para la proyección de esta modalidad; la Operativa donde se presentan los resultados y por último la fase de Control, que presupone el implemento de un plan de acción para la retroalimentación y seguimiento en función de la mejora continua en la implementación del procedimiento.(Peñalver, 2015)

\section{Fase: Organizativa}

Paso 1 Formación del grupo de trabajo

El grupo de trabajo se integró por el equipo de investigación, comercial del MINTUR Villa Clara, Cienfuegos y Sancti Spíritus, representantes del gobierno local, especialistas en la actividad de eventos: profesores de la Universidad Central Marta Abreu de las Villas (UCLV) y Formatur de las 3 provincias centrales.

Paso 2 Elaboración del cronograma de trabajo

El período de trabajo para desarrollar, validar y aplicar el procedimiento propuesto estuvo comprendido entre el 1 de junio de 2015 y el 15 de diciembre de 2017, período en el que se seleccionó, se ordenó la información recopilada con antelación y se aplicaron las herramientas necesarias para complementar el estudio. El cronograma comprende las fases, etapas y pasos del procedimiento, destaca como responsable de su implementación a la investigadora y acuerda sesiones y encuentros mensuales entre los miembros del grupo de trabajo.

\section{Fase: Analítica. Diagnóstico de la gestión del Turismo de Eventos e Incentivos.}

El diagnóstico estratégico fue desarrollado a partir del procedimiento aportado por Bermúdez (2013) puesto que, metodológicamente, se ajusta a las necesidades de la presente investigación, además de que da los aportes necesarios que sirven de antecedentes para plantear la estrategia de gestión del producto eventos e incentivos.

La aplicación del procedimiento permitió llegar a un conjunto de resultados de diagnóstico:

En el ámbito nacional los principales destinos competidores para la zona central son La Habana, Matanzas y Santiago de Cuba, que han sido históricamente los destinos más fuertes en la realización de eventos; no obstante, se consideran competidores todos los destinos de Cuba, pero desde una visión más moderna, representan posibles alianzas para la creación de un producto integrado. 
Según trabajo de campo realizado en instalaciones de Gaviota, ha aumentado significativamente en los últimos años, la afluencia de canadienses al destino Cayos de Villa Clara, considerado actualmente el escenario preferido para la celebración de bodas, específicamente para canadienses. Durante la temporada alta se realizan aproximadamente cinco bodas semanales en los hoteles de la Cayería Norte, fundamentalmente.

En el resto de las instalaciones del destino central de Cuba el principal mercado que asiste a eventos ha sido Francia, seguido de Alemania, Holanda y Reino Unido, además de cubanos autofinanciados, seguidos de Venezuela.

La inexistencia de información relativa a eventos registrada es latente en Gaviota, el MINTUR, así como en los propios hoteles sedes y sedes de eventos.

En el destino se ha partido del criterio erróneo de considerar que los eventos se trabajan como cualquier otra modalidad, sin comprender que requiere de la alianza con instituciones ajenas al sector turístico, sin las cuales es imposible desarrollarla, existiendo un divorcio entre sociedades científicas, MINTUR, comité organizador, sedes de eventos, entidades de servicios, además de que no existen OPC en el destino.

De manera general la oferta en el territorio se ha mantenido estática, poco diversificada y con falta de integración y débil gestión. Los mayores crecimientos se producen en el grupo Gaviota sobre todo por la apertura acelerada de capacidades hoteleras en la Cayería Norte y altos niveles de comercialización, mientras que el crecimiento de entidades del MINTUR es muy discreto.

Por otra parte, el precio; se utiliza el sistema de costos estándar de manera inflexible y no hay ninguna ingeniería de precios. Para fijar el precio se le añade al costo un margen de utilidad según la instalación que comercialice, siendo más apropiado combinar en el momento de fijar el precio lo que están dispuesto a pagar los eventistas y la media de la competencia.

Aunque lo más alarmante hasta el momento es la relación calidad/precio, que se ha visto afectada, según encuestas realizadas por la Oficina de Información Turística (Infotur) en la zona central, por condiciones en la infraestructura y en servicios de alimentos y bebidas en las instalaciones sedes de eventos del MINTUR principalmente; mientras que la competencia con hoteles sedes de Gaviota y la nueva competencia con los cuentapropistas, en el tema calidad/precio, sí adquiere una dimensión importante, pues conocen muy bien su punto de equilibrio.

El levantamiento de recursos desarrollado trasluce la existencia de numerosas posibilidades, muchas de ellas bajo poca o ninguna explotación. En lo relativo a la infraestructura, se cuenta con una planta hotelera fundamentalmente administrada por el Grupo Gaviota S.A., con varias instalaciones hoteleras de categoría 4 y 5 estrellas que suman más de 30000 capacidades habitacionales; marina, agencia de viajes y transporte turístico. En la zona central se refuerza la actividad de alojamiento con las casas particulares.

A pesar de realizarse diferentes eventos y demás actividades en las principales sedes del destino, se evidencia que el enfoque de los objetivos no está en función de una gestión 
basada en la integración del producto eventos al producto turístico del centro de Cuba, lo que trae desventajas para el posicionamiento del mismo en el mercado de eventos a nivel internacional y nacional.

Sedes de eventos e incentivos reconocidas en el destino, están fuera del sistema de turismo: el Centro de Convenciones Bolívar pertenece al Ministerio de la Agricultura; el Centro de Negocios pertenece a las Fuerzas Armadas Revolucionarias; Expocentro pertenece al Poder Popular, por lo que su gestión es aislada y no controlada adecuadamente, lo cual impide y limita la adecuación a requisitos, normas y legislaciones establecidas por la ICCA y dictadas por el BCC, certificadas a partir de las normas DIN 15906.

A partir de un estudio del plan de capacitación de las instalaciones vinculadas a la realización de eventos, se infiere que ninguna posee entre sus proyecciones la formación de un personal calificado en la especialidad. Una de las acciones que ha detenido el crecimiento profesional de los OPC ha sido la desaparición de la Cátedra de Gestión de Eventos en Formatur y junto a ello los programas de formación de OPC y de otros perfiles profesionales y cursos de maestrías y diplomados auspiciados por la Escuela de altos Estudios de Hotelería y Turismo en el Hotel Sevilla, que se impartían para la formación profesional en el país.

En lo relativo a la variable Comunicación, no se explotan todas las alternativas para la difusión de la información referente a los eventos, provocando que esta no llegue a todos los posibles públicos. Los especialistas opinan que las causas fundamentales que originan esta situación están dadas por la inexistencia de un presupuesto que posibilite cubrir la operación.

El mercado generador de los eventos más importantes en el territorio incluye la UCLV, el Instituto de Biotecnología de las Plantas, la Universidad de Ciencias Médicas, el Ministerio de Agricultura, la Cámara de Comercio de Villa Clara, la Universidad Pedagógica y del Deporte. Dichos organismos son reconocidos a nivel nacional por su enorme potencial científico, y generan eventos de gran importancia y actualidad a nivel mundial.

Como parte del diagnóstico se desarrolla un análisis DAFO, que concluye en que la gestión de la modalidad Eventos e Incentivos es un negocio incógnito en el destino, pues se encuentra en el cuadrante donde se interceptan las debilidades y las oportunidades, que implica el trazarse una estrategia adaptativa o de reorientación.

\section{Fase: Estratégica. Gestión del Turismo de Eventos e Incentivos.}

De la Matriz DAFO se puede definir el problema estratégico que se presenta en el destino. En especial todas las debilidades tienen una significación negativa en el desarrollo de eventos e incentivos en el territorio, pero el principal problema es la gestión ineficaz de esta modalidad, sin tener en cuenta el carácter sistémico e integrador del turismo en el destino ni la importancia de la comercialización de los eventos para el equilibrio de la actividad turística y como creador de una imagen de destino turístico atractivo, integrado y armónico.

Etapa 5. Definición del propósito estratégico 
Paso 13. Definición de la Misión.

Somos un destino turístico emergente en el desarrollo de eventos e incentivos, que promueve, gestiona, comercializa y acoge en sus sedes estas actividades en diferentes tipologías, contando con infraestructura, tecnología, Organizadores Profesionales de Congresos y capital humano especializado en servicios que garantizan el éxito de los eventos y la satisfacción de los eventistas y acompañantes, todo ello integrado en un programa de eventos y de actividades de ocio para el disfrute pleno del producto turístico.

Paso 14. Definición de la Visión.

Ser el destino turístico líder en el desarrollo de Eventos e Incentivos en Cuba, garantizando la efectividad de la comercialización y la gestión de eventos, infraestructura, tecnología y servicios, con la profesionalidad y especialización que la modalidad requiere.

Paso 15. Valores compartidos

Los valores determinan el comportamiento requerido para el logro de los objetivos planteados es así que se requiere de: Ética y moral revolucionaria, Profesionalidad, Productividad, Trabajo en equipo, Capacidad de cambio, Creatividad.

Paso 16. Proyección de escenarios

\begin{tabular}{|c|c|c|}
\hline Escenario Negativo & Escenario Positivo & Escenario más probable \\
\hline $\begin{array}{l}\text { 1. Se han producido las } \\
\text { condiciones que han } \\
\text { llevado al país a pasar a la } \\
\text { primera etapa prevista en } \\
\text { los planes de defensa. } \\
\text { 2. Se ve interrumpido } \\
\text { totalmente el flujo de } \\
\text { remesas y viajes hacia } \\
\text { Cuba desde los E.U.A. } \\
\text { 3. Se agudiza el efecto de } \\
\text { la crisis económica } \\
\text { mundial en la economía } \\
\text { nacional. } \\
\text { 4. Se estanca o disminuye } \\
\text { la afluencia de turismo } \\
\text { internacional en el país. } \\
\text { 5. Se destruyen y dañan } \\
\text { varias instalaciones sedes } \\
\text { de eventos a consecuencia } \\
\text { de fenómenos } \\
\text { climatológicos, que causan } \\
\text { graves pérdidas } \\
\text { financieras/económicas. } \\
\text { 6. Dificultad para } \\
\text { mantener los } \\
\text { requerimientos en }\end{array}$ & $\begin{array}{l}\text { 1. Se elimina el bloqueo } \\
\text { económico, siendo posibles } \\
\text { las relaciones comerciales, } \\
\text { intercambio y colaboración } \\
\text { entre EE.UU. y Cuba. } \\
\text { 2. La economía del país logra } \\
\text { incrementos sustanciales en } \\
\text { su PIB y otros indicadores } \\
\text { económicos. } \\
\text { 3. Aumentan } \\
\text { significativamente los } \\
\text { niveles de afluencia de } \\
\text { turismo internacional en el } \\
\text { destino. } \\
\text { 4. Aumento del } \\
\text { financiamiento para } \\
\text { infraestructura, tecnología y } \\
\text { servicios en sedes de eventos } \\
\text { del destino } \\
\text { 5. Existe un aumento de las } \\
\text { oportunidades de negocio, } \\
\text { sobre todo en el ámbito } \\
\text { nacional, y las exportaciones, } \\
\text { que pueden ser aprovechadas } \\
\text { para la proliferación de } \\
\text { reuniones. }\end{array}$ & $\begin{array}{l}\text { 1. Se mantienen las } \\
\text { condiciones del bloqueo y } \\
\text { continúan controlados los } \\
\text { viajes procedentes de los } \\
\text { E.U.A, impidiendo } \\
\text { aprovechar ese mercado de } \\
\text { eventos. } \\
\text { 2. Se mantienen los niveles } \\
\text { de afluencia de turismo. } \\
\text { 3. Se toman medidas para } \\
\text { proteger las infraestructuras } \\
\text { para eventos ante } \\
\text { fenómenos climatológicos. } \\
\text { 4. Se incrementan los } \\
\text { convenios de colaboración } \\
\text { con los países del ALBA, } \\
\text { la CELAC y Asia, posibles } \\
\text { mercados turísticos a } \\
\text { explorar. } \\
\text { 5. Se percibe un } \\
\text { crecimiento discreto de la } \\
\text { economía cubana. } \\
\text { 6. Se mantiene la } \\
\text { estabilidad política y social, } \\
\text { aunque se prevé cierto }\end{array}$ \\
\hline
\end{tabular}




\begin{tabular}{|l|l|l|}
\hline infraestructura y & 6. Se fortalece la gestión y la & incremento en la actividad \\
tecnología para eventos. & comercialización del & delictiva. \\
7. Marcado & producto turístico o, con & 7. Se mantiene el turismo \\
posicionamiento de & visión de destino integrado. & como motor de la economía \\
algunos destinos & 7. Crece la competencia y la & y se abren nuevas \\
competidores en la & seriedad de las relaciones & oportunidades para el \\
realización de eventos. & entre prestatario y cliente, & desarrollo de los eventos en \\
8. Deficiente & mejorando la calidad en los & los destinos. \\
comercialización del & servicios turísticos a nivel de & 8. Incremento sostenido de \\
Turismo de Eventos a & destino. & la demanda turística en los \\
nivel de país. & 8. Auge de programas de & hoteles de la Cayería Norte \\
9. Se reduce & formación y capacitación & y proliferación de eventos, \\
significativamente el & para eventos por parte del & negocios y reuniones en los \\
mercado del producto & MINTUR y Formatur, así & mismos. \\
eventos a nivel nacional e & como el fortalecimiento de & 9. Aumentan las ofertas y \\
internacional. & los comités organizadores y & la calidad del producto \\
10. Deterioro progresivo la & de los OPC. & Eventos e Incentivos. \\
imagen del destino en la & 9. Alto nivel científico, & 10. Se flexibiliza la política \\
realización de eventos por & cultural y la presencia de & de centralizar las decisiones \\
improvisaciones en la & prestigiosos investigadores & comerciales, por lo que el \\
gestión y & en el destino, que favorecen & destino gana autonomía \\
comercialización. & el desarrollo de eventos de & para el desarrollo del \\
diferentes temáticas & producto turístico Eventos \\
& 10. Aumento significativo de & Incentivos. \\
cuotas de mercado de & 11. Se avanza en el \\
eventos en el destino, en & perfeccionamiento de la \\
gestión y comercialización \\
del turismo de eventos \\
especial en las sedes de la & Cayería Norte. & turístico. \\
& & \\
& & \\
& &
\end{tabular}

Paso 17. Formulación estratégica del turismo de Eventos e Incentivos en el destino turístico.

La estrategia general del destino para desarrollar al máximo el turismo de eventos e incentivos, está encaminada a: lograr la efectividad de la gestión del turismo de eventos e incentivos con la integración de todos los actores y gestores de la actividad, el MINTUR, Gaviota, sedes de eventos y sedes generadoras y el restablecimiento del BCC como entidad clave en la gestión de eventos; potenciando el alto nivel científico, cultural y la presencia de prestigiosos investigadores en el destino, garantizando la infraestructura, tecnología y servicios a eventos, la profesionalidad y especialización del personal y OPC, además del aprovechamiento de los recursos y atractivos turísticos del destino para brindar una oferta complementaria de lujo, permitiendo consolidar este producto turístico e insertarse exitosamente en el mercado de la modalidad y así contrarrestar el marcado posicionamiento de algunos destinos competidores en la realización de eventos para aprovechar la oportunidad que representa el incremento del mercado de eventos, tanto a nivel nacional como internacional. 
Etapa 6. Proyección estratégica

Paso 18. Determinación de las áreas de resultados clave (ARC). Paso 19. Determinación de objetivos estratégicos por ARC y estrategias por Objetivos

\section{Gestión de eventos}

Objetivo \# 1. Velar por parte del MINTUR a partir del 2018, el cumplimiento de las normas internacionales y nacionales para la organización y realización de eventos en las sedes, así como las políticas de desarrollo turístico adecuadas al contexto del turismo de eventos en el territorio.

Estrategias:

1. Implementar en las sedes, procedimientos, metodologías y normas para la organización de eventos, que garanticen un desarrollo exitoso de los mismos.

2. Seleccionar el comité organizador de cada evento de forma representativa e integradora, que incluya miembros de la sede del evento y de la que lo genera, el MINTUR y un especialista en organización profesional de eventos.

3. Preparar el evento con suficiente antelación para planificar con tiempo las actividades de comunicación, promoción, así como su venta al mercado. Realizar por parte de cada sede el calendario de eventos propios de carácter nacional e internacional y enviarlo al OPC del MINTUR.

4. Definir por parte del OPC y comité organizador de los eventos en las sedes, las temáticas de los mismos, duración, la confección de paquetes con precio de la cuota de inscripción y otros servicios, la tecnología necesaria y los servicios a eventos y complementarios adecuados.

5. Definir los medios de comunicación promocional para los eventos.

6. Definir las sesiones de trabajo y dar el adecuado seguimiento a la evolución de las actividades de gestión.

7. Elaborar una base de datos y actualizarla periódicamente, permitiendo el estudio y análisis de la actividad de eventos en beneficio de la gestión de la misma.

\section{Gestión de incentivos}

Objetivo \# 2. Realizar un estudio en el destino (encuestas, entrevistas, declaraciones a la llegada al aeropuerto) enfocado a determinar el número mensual de visitas por incentivos y explorar sus necesidades y expectativas

para crear un producto que satisfaga este segmento y lanzarlo a los mercados correspondientes.

Estrategias:

1. Crear un equipo de trabajo con el fin de realizar el estudio en el 2020.

2. Diseñar una entrevista sencilla, en inglés y francés, y aplicarla a la llegada de los visitantes al aeropuerto. 
3. Determinar aleatoriamente los meses que va aplicarse y tomar un tamaño de muestra representativo de los principales mercados emisores.

4. Según los resultados de su aplicación, determinar la viabilidad de crear un paquete exclusivo para este segmento de mercado.

\section{Gestión de sedes}

Objetivo \# 3. Lograr paulatinamente los niveles estándar de excelencia de la infraestructura para eventos, acorde a las normas internacionales y nacionales.

Estrategias:

1. Actualizar el inventario de las sedes de eventos y sus condiciones y características: muebles, climatización, y si disponen de impresoras, escáner, fotocopiadoras, internet, fax y medios audiovisuales, entre otros.

2. Mejorar, en tanto sea posible y según las necesidades para su desempeño, la disponibilidad de las sedes, sea o no una entidad del sistema de turismo.

Objetivo \# 4. Garantizar por parte del MINTUR, las facilidades, equipamientos y tecnología y el correcto funcionamiento del sistema informativo en instalaciones y sedes del destino, de manera que puedan efectuarse exitosamente los eventos e incentivos en el territorio a partir de 2018.

Estrategias:

1. Evaluar frecuentemente el estado técnico de las facilidades y tecnología disponibles en las sedes.

2. Fomentar el trabajo en equipo entre las sedes, de manera que las posibilidades de unas complementen las dificultades de otra y a la postre se desarrolle el producto Eventos e Incentivos.

3. Considerar la contratación a particulares que puedan brindar sus aportes en cuanto a tecnología y operaciones si fuera necesario.

4. Establecer el envío al MINTUR de un reporte estadístico de los eventos realizados en las sedes, incluyendo un breve resumen del evento, tipología, fecha en que se realiza, OPC, comité organizador, así como un resumen de una encuesta de satisfacción a los participantes, además de los indicadores propios de la modalidad.

\section{Gestión de los Recursos Humanos}

Objetivo \# 5. Convocar por parte del MINTUR y Formatur cursos que respondan a las necesidades de formación o capacitación de directivos y trabajadores vinculados a la actividad de eventos, así como de OPC para ofertarlos a partir del 2018.

Estrategias:

1. Valorar la posibilidad de formar OPC en el territorio, y convocar a un curso especial para tener un profesional especializado en la realización de eventos que responderá a las nuevas tendencias del negocio. 
2. Incluir dentro del staff del MINTUR un Especialista en eventos u OPC que garantice la profesionalidad y la calidad en la gestión y el desarrollo de los eventos en el territorio.

3. Realizar un Diagnóstico de las Necesidades de Aprendizaje (DNA) que tengan los directivos y trabajadores vinculados a la actividad de eventos en las sedes, respecto a la misma.

4. Verificar que existan en FORMATUR los cursos para la capacitación que demande el DNA.

5. Garantizar que el personal directamente involucrado en la realización de eventos en las sedes reciba la actualización necesaria en su formación.

\section{Gestión económico-financiera}

Objetivo \# 6. Establecer, por parte de la dirección económica de las sedes de eventos, la política financiera en las mismas, según las regulaciones del MEP e incluyendo la planificación del presupuesto de operación y un mecanismo de control efectivo a través de la evaluación de indicadores económicos en los próximos 5 años.

Estrategias:

1. Diseñar en cada sede, una política financiera acorde a lo establecido por el MEP y sus propias características como entidad.

2. Flexibilizar los plazos, formas de pago, y otros trámites para que los eventistas abonen su cuota de inscripción.

3. Velar por el cumplimiento de los cobros y pagos, respetando la contratación.

4. Establecer un mecanismo para el control económico-financiero y retroalimentación a la futura planificación de los recursos de las sedes.

5. Valorar el estado actual de la infraestructura física y de servicios para eventos e incluir un estudio de factibilidad para la aprobación de una futura inversión en las sedes requeridas.

6. Estudiar la posibilidad de autofinanciamiento de las sedes.

\section{Gestión de sedes generadoras de eventos}

Objetivo \#7. Integrar las sedes del destino turístico, teniendo como rector el MINTUR en tanto las Secretarias de Eventos no se restablezcan, de modo que se trabaje en equipo para lograr el desarrollo exitoso de los eventos.

Estrategias:

1. Mantener actualizado en el MINTUR un inventario de todas las sedes generadoras y aquellas que pudieran generar eventos y sus características

2. Mantener actualizados en el MINTUR, los contactos de cada sede.

3. Realizar por parte de cada sede, el calendario de eventos propios de carácter nacional e internacional y enviarlo al MINTUR. 


\section{Gestión de la comercialización}

Objetivo \# 8. Definir, por parte del MINTUR, la estrategia comercial del producto eventos e incentivos en Villa Clara, de modo que se avance hacia una comercialización efectiva a partir del 2018.

\section{Estrategias:}

1. Identificar el mercado, la competencia y el producto del destino, y de ahí, el público objetivo al que se dirige el producto, las ventajas competitivas del destino respecto a otros y los atractivos de posicionamiento.

2. Establecer una política de contratación efectiva para los próximos 5 años, teniendo en cuenta los principales segmentos de mercado de eventos que se pueden captar.

3. Preparar grupos FAM con representación de las principales agencias de viajes que comercializan los eventos del territorio para presentarles las facilidades disponibles y los servicios especializados de que se dispone en las sedes.

4. Incluir, dentro de la página Web de Infotur en Villa Clara, las ofertas que ofrece en materia de eventos y las facilidades, infraestructura y servicios con que cuenta el destino.

5. Definir por parte del departamento comercial del MINTUR y cada sede un plan de Marketing acorde al estudio de la modalidad de eventos.

6. Conformar el Producto que distingue el destino e individualmente cada sede, para tener identificadas las acciones en cuanto a precio y definir cómo va a ser más efectiva la distribución al mercado.

7. Estudiar la competencia para poder determinar nuevos elementos diferenciadores que permitan el diseño de un producto más competitivo.

8. Realizar estudios de mercado para evaluar constantemente las posibilidades de atracción de nuevos clientes creando ofertas especiales.

9. Desarrollar estrategias de marketing electrónico acorde a las tendencias más actuales en las que se mueve el mercado.

\section{Gestión de la comunicación promocional}

Objetivo \#9. Realizar un plan de Comunicación para eventos e incentivos a nivel de destino por parte del MINTUR y a nivel individual de cada sede por parte de sus direcciones, que permita definir a partir del 2018, las acciones específicas del MIX de Comunicación.

Estrategias:

1. Gestionar un presupuesto para la comunicación de las actividades y eventos que potencia el destino/sede.

2. Diseñar una campaña de publicidad para los eventos internacionales y aquellos nacionales más importantes. 
3. Definir el momento clave para la promoción de ventas de los eventos y cómo los eventistas tendrán acceso a las ventas directas.

4. Determinar las actividades de Merchandising y Marketing directo.

5. Potenciar el trabajo con la modalidad de los Community Managers en el destino.

\section{Gestión de la Calidad de los servicios a eventos}

Objetivo \# 10. Incluir, como parte del sistema de Gestión de la Calidad en cada sede, cómo se llevará a cabo la misma para la actividad de eventos, según las normas previamente establecidas por el MINTUR, y demás organismos internacionales rectores de la actividad de eventos.

\section{Estrategias:}

1. Asegurar el diseño e implementación del Sistema de Gestión de la Calidad en cada sede.

2. Evaluar el cumplimiento de las medidas que establecerá el sistema de gestión para garantizar la calidad.

3. Establecer como parte de la gestión de la calidad, un sistema de encuestas que permitan evaluar constantemente la calidad de los servicios a eventos en las sedes del destino.

\section{Gestión de actividades complementarias}

Objetivo \# 11. Crear una oferta complementaria a la realización de eventos, aprovechando las potencialidades del destino, basada en la integración de productos y servicios turísticos variados que enriquezcan la experiencia tanto para eventistas como para acompañantes.

Estrategias:

1. Diseñar una cartera de productos a ofertar para eventos, basado en el inventario de recursos y atractivos turísticos del destino que se incluye más arriba, y según las características del evento, su duración y tipología: visita a la Ciudad de Santa Clara o Remedios, visita a las Playas de la Cayería Norte; Visitas a Cienfuegos y Rancho Luna o Trinidad; Paseo a la montaña, u otras actividades culturales y recreativas como galas, presentaciones, conciertos, fiestas, entre otras.

2. Hacer una ficha de costo por cada actividad en las sedes.

3. Establecer precio de cada actividad.

4. Conformar paquetes de servicios con flexibilidad en las opciones, hasta llegar a la medida de eventos y eventistas.

\section{Fase: Operativa. Presentación de los resultados}

Paso 20. Confección del informe final

El presente informe cumple con la presentación del procedimiento y su aplicación en la totalidad de sus fases, etapas y desarrollo, con especial énfasis en el diagnóstico de la gestión del turismo de eventos e incentivos en el destino, cuyos resultados se concretan 
en la fase estratégica, que da vida a la estrategia de gestión de eventos e incentivos definida para el destino y su proyección estratégica a modo de objetivos por ARC y estrategias por objetivos.

\section{Fase: Control.}

Paso 21. Control, retroalimentación y seguimiento

Para la retroalimentación y el seguimiento de la aplicación del procedimiento en el destino, los expertos recomiendan al equipo de trabajo diseñar un Plan de acción para garantizar el monitoreo del mismo y adoptar las medidas correctoras necesarias según la experiencia práctica, en las condiciones y el momento que el destino demande:

- Aplicar el procedimiento al menos 2 veces para evaluar desviaciones en los resultados.

- Aplicar anualmente el diagnóstico de la gestión de eventos e incentivos en el destino para hacer análisis comparativos tanto cualitativos como cuantitativos en función de los indicadores del turismo de eventos mencionados en la Etapa 2 del diagnóstico.

- Evaluar el cumplimiento de las estrategias y objetivos de gestión propuestos y el progreso de la modalidad de eventos en el destino.

- Analizar los elementos que han cambiado

- Actualizar el procedimiento y hacer ajustes necesarios a la estrategia

\section{Conclusiones}

La aplicación del procedimiento seleccionado para el diagnóstico estratégico de la modalidad en la zona central del país, permitió indagar en las deficiencias encontradas dentro de su gestión, a la par de identificar una serie de fortalezas y oportunidades sin explotar, debidos en gran medida a la existencia de una gestión empírica y pobremente orientada al Turismo de Eventos e Incentivos.

> La implementación del procedimiento para la proyección de la modalidad, toma como bases los resultados arrojados por el diagnóstico previo, y deviene en una estrategia orientada a maximizar las fortalezas con que cuenta el destino, identificada con sus condiciones y características peculiares, dotando así a la zona central de una herramienta eficaz y eficiente para la gestión estratégica del Turismo de Eventos e Incentivos. 


\section{Referencias Bibliográficas}

Bermúdez, (2013). Procedimiento para el diagnóstico de la gestión del turismo de eventos e incentivos en el destino turístico Villa Clara (Tesis de Maestría en Gestión Turística). Universidad Central Marta Abreu de Las Villas, Santa Clara, Villa Clara, Cuba.

International Congress and Conventions Association. (2017). ICCA Statistics Report 2016. Recuperado de http://www.iccaworld.com/.

Ministerio de Turismo de Cuba, (2011) Resultados del sector en 2011 y perspectivas para el año próximo. Cuba. MINTUR La Habana.

Morales, B. R. S. (2014). Organización de Congresos y Eventos. Organización y Clasificación De Eventos. Universidad Alas Peruanas.

Organización Mundial del Turismo, (OMT). (2016). Panorama OMT del turismo internacional, 2016. Recuperado en http://www.worldtourism.org/.

Partido Comunista de Cuba, (PCC). (2016). Actualización de los Lineamientos de la Política Económica y Social del Partido y la Revolución para el período 20162021. Séptimo Congreso del Partido.

Peñalver, Y., (2015) Procedimiento para la gestión del turismo de eventos e incentivos. Aplicación en el destino turístico Villa Clara. (Tesis de Maestría en Gestión Turística). Universidad Central Marta Abreu de Las Villas, Santa Clara, Cuba

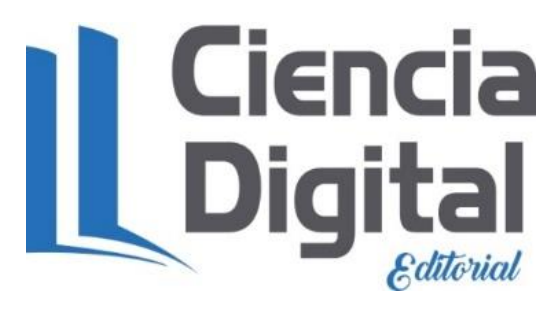


Para citar el artículo indexado.

Taboada K. \& Campos L. (2017). Estrategia de proyección del turismo de eventos e incentivos en la zona central de Cuba. Revista electrónica Explorador Digital 1(2), 4358. Recuperado desde:

http://cienciadigital.org/revistacienciadigital2/index.php/exploradordigital/article/view/321/7 $\underline{33}$

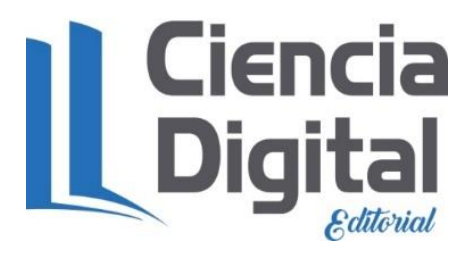

El artículo que se publica es de exclusiva responsabilidad de los autores y no necesariamente reflejan el pensamiento de la Revista Explorador Digital.

El articulo queda en propiedad de la revista y, por tanto, su publicación parcial y/o total en otro medio tiene que ser autorizado por el director o editor de la Revista Explorador Digital.
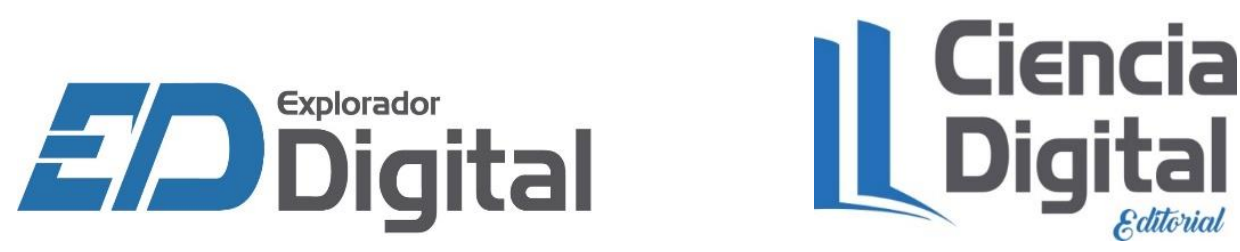